\title{
Empilhamento i-CRS: uma nova alternativa para o imageamento sísmico
}

Dheymison Alves Moreira ${ }^{1}$ e Pedro Chira-Oliva ${ }^{2 \star}$

1 Faculdade de Geofísica (FAGEOF)/Universidade Federal do Pará.

2 Instituto de Estudos Costeiros( IECOS) /Universidade Federal do Pará, *e-mail: chira@ufpa.br

Copyright 2014, SBGf - Sociedade Brasileira de Geofísica

Este texto foi preparado para a apresentação no VI Simpósio Brasileiro de Geofísica, Porto Alegre, 14 a 16 de outubro de 2014. Seu conteúdo foi revisado pelo Comitê Técnico do VI SimBGf, mas não necessariamente representa a opinião da SBGf ou de seus associados. É proibida a reprodução total ou parcial deste material para propósitos comerciais sem prévia autorização da SBGf.

\section{Resumo}

Over the past years, multiparameter traveltime approximations have been introduced in order to improve the limitations of the CMP stacking technique. This technique considers a single parameter (i.e. NMO velocity), where the stacking approximation takes into account the seismic common-midpoint (CMP) configuration. These new methods are characterized by considering more than one parameter and their approximations describe the traveltime surface for a reflected event in the short offset limit. Recently, been introduced a new non-hyperbolic traveltime approximation known as implicit-Common-Reflection-Surface (i-CRS). It was derived from Snell's law for a spherical interface and leads to an implicit expression for the traveltime surface. This new approximation can be applied for monotypic waves in isotropic media and considers seismic anisotropy. In this paper, we applied the i-CRS traveltime approximation on synthetic datasets to verify the performance of the same in the simulation of Zero-Offset (ZO) sections and the recovery of seismic traces compared with conventional stacking methods.

\section{Introdução}

Os tempos de trânsito das ondas elásticas são o ingrediente chave em qualquer etapa do processamento de dados sísmicos. Primeiramente, as expressões analíticas são usadas durante o empilhamento para obter a primeira imagem. No final da cadeia de processamento, após a construção do modelo de velocidade ser carregado neste processamento, os tempos de trânsitos são gerados numericamente para o modelo ser usado na migração final pré-empilhada para a imagem final. $O$ principal objetivo do método sísmico é obter uma imagem da subsuperfície a partir do levantamento de dados sísmicos, sejam sintéticos ou reais.

Devido ao fato de que em um experimento sísmico as fontes e receptores podem somente serem distribuídas na subsuperfície da terra ou próximo dela, tem que depender ou contar com a energia refletida, difratada ou criticamente refratada para fornecer esta imagem.

Melhorar a qualidade das seções sísmicas é muito importante e relevante para a interpretação sísmica, e.g. na localização de falhas geológicas, estruturas em forma de cunha, flancos de domos ou indicar possível presença de saltos ou terminações de soleiras.
Após um levantamento sísmico dispomos de uma grande quantidade de dados que muita das vezes é redundante. Um caminho conveniente para reduzir eficientemente esta redundância é empilhar todos os traços que carregam mais ou menos a mesma informação.

O processo de empilhamento sísmico simula uma seção $\mathrm{ZO}$ e fornece pela primeira vez uma imagem da subsuperfície. Um dos métodos mais frequentemente aplicados é o clássico empilhamento Common-midpoint, CMP (MAYNE, 1962). Durante este empilhamento, a energia coerente de todos os traços dentro de uma configuração fonte-receptor CMP é somada, resultando em um traço empilhado. Este método apresenta limitações no empilhamento, pois o mesmo é realizado ao longo de uma única curva de tempo de trânsito e depende de um único parâmetro, a velocidade Normalmoveout (NMO).

O empilhamento sísmico multiparamétrico tem-se tornado, portanto, uma ferramenta muito importante para o processamento de dados de reflexão sísmica. Ao longo dos últimos anos, diversas aproximações de tempos de trânsito multiparamétricos foram apresentadas como uma extensão da técnica de empilhamento sísmico convencional CMP. Entre os principais exemplos destas aproximações destacam a aproximação da hipérbole recortada (de Bazelaire, 1988), Common-ReflectionSurface (CRS, Jäger et al., 2001, Müller, 1999), Multifoco (MF, Landa et al., 1999, Landa et al, 2010) entre outras aproximações.

As aproximações de tempos de trânsito mencionados no parágrafo anterior descrevem a superfície de tempos de trânsito para um evento refletido no intervalo de um afastamento fonte-receptor curto. A exatidão destes métodos difere e depende não somente do afastamento fonte-receptor considerado, sendo também da curvatura do refletor.

A qualidade dos resultados do empilhamento depende fundamentalmente da exatidão da aproximação de tempos de trânsito utilizado no empilhamento. As aproximações de tempo de trânsito hiperbólicas com função de afastamento são exatas para o caso de um refletor plano com mergulho na subsuperfície. Qualquer desvio desta suposição resulta em uma aproximação de tempo de trânsito não hiperbólico. As principais causas para isto podem ser a heterogeneidade vertical e/ou lateral da subsuperfície em um meio anisotrópico não elíptico ou a forma de refletor mesmo, ou seja, sua curvatura (Fomel e Grechka, 2001).

Geralmente, as aproximações baseadas nas expressões contendo duplas raízes quadradas, como o método Multifoco são mais adequadas para a descrição dos tempos de trânsito de difrações que as formulas hiperbólicas. Apesar de que, a fórmula Multifoco é mais 
adequada que a aproximação do tempo de trânsito CRS convencional para a descrição das difrações, nenhuma delas considera a anisotropia sísmica (Vanelle et al, 2010).

Com a finalidade de tentar suprir as limitações do CRS em relação às difrações e anisotropia, Vanelle et al (2010) introduziu uma aproximação de tempos de trânsito para ondas monotípicas. Esta aproximação foi obtida a partir da Lei de Snell para uma interface esférica que nos leva a uma expressão implícita para a superfície de tempos de trânsito. Pode ser considerada como uma representação baseado no modelo da teoria do método CRS. Como consequência disto e de sua natureza implícita é referida como a aproximação i-CRS. Eles demonstraram o potencial de ajuste desta aproximação para o caso da velocidade constante. Esta fórmula CRS implícita foi introduzida como uma alternativa de aproximação de tempos de trânsito não-hiperbólico que intrinsecamente explique melhor a curvatura do refletor, não somente no ponto médio, mas também na direção do afastamento.

Vanelle et al. (2012) aplicaram a anterior aproximação em meios isotrópicos e anisotrópicos. Os resultados têm demonstrado uma exatidão superior quando comparados com o método CRS convencional para uma grande variedade de formas de refletores desde refletores planos até formas mais complexas com características de gerar eventos de difrações.

Em virtude das vantagens do método CRS implícita, é que será aplicado este método em dados sintéticos com a finalidade de testar sua eficácia na simulação de seções sísmicas de afastamento-nulo considerando eventos de reflexão e comparar a mesma com o método CRS convencional até a quarta-ordem.

\section{Método \\ Vanelle et al. (2010) apresentaram uma nova aproximação de tempos de trânsito de empilhamento para ondas monotipicas em meio isotrópicos referindo-se como a aproximação common-reflection-surface ou superfície de reflexão comum implícita (i-CRS). \\ Tanto Vanelle et al. (2010) e Schwarz (2011) introduziram com esta aproximação i-CRS uma aproximação muito razoável para explicar melhor a curvatura do refletor assumindo um refletor circular. Esta derivação está baseada na idéia de Boris Kashtan (Comunicação Pessoal) repassada para estes autores ao levar em consideração uma nova aproximação implícita para avaliar o ponto de reflexão sobre um círculo fazendo uso do Principio de Fermat.}

Vanelle et al. (2011) consideraram um refletor esférico em um meio homogêneo. O raio do reflector é $\mathrm{R}$, com o seu centro no local $\left(x_{c}, 0, H\right)$, como mostrado na Figura 1. $x_{1}$ e $x_{2}$ são coordenadas de uma fonte e de um receptor, respectivamente, ambos na profundidade $z=0$ e $y=0$. O ângulo $\theta$ define o ponto de reflexão em $\vec{r}=(R \operatorname{sen} \theta, 0, H-R \cos \theta)$. As velocidades do raio/grupo dos segmentos do raio de ida e volta são $v_{i}\left(\vartheta_{i}\right)$, com os âgulos grupo $\vartheta_{i}$.
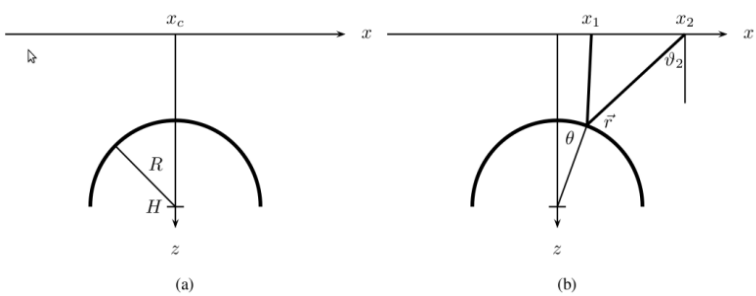

Figura 1 (a) - Geometria do refletor e b) aquisição para um refletor esférico. O ponto de reflexão $\vec{r}$ é definido pelo ângulo $\theta$ (Vanelle et al., 2012).

\section{Aproximação i-CRS}

Considerando a Figura 1, temos que os tempos de trânsito $t_{i}$ dos segmentos de raios de ida e volta expressos dados por:

$$
\begin{aligned}
& t_{1}^{2} \\
& =\frac{\left(\Delta x_{m}-h-\Delta x_{c}-R \operatorname{sen} \theta\right)^{2}+(H-R \cos \theta)^{2}}{v_{1}^{2}\left(\vartheta_{1}\right)} \\
& t_{2}^{2} \\
& =\frac{\left(\Delta x_{m}+h-\Delta x_{c}-R \operatorname{sen} \theta\right)^{2}+(H-R \cos \theta)^{2}}{v_{1}^{2}\left(\vartheta_{1}\right)}
\end{aligned}
$$

sendo:

$$
t=t_{1}+t_{2}
$$

A aproximação i-CRS também pode ser expressa em função dos três parâmetros do CRS convencionais: a) o ângulo de emergência do raio de reflexão normal em relação à normal da linha de medição $\left(\beta_{0}\right)$, b) a curvatura da onda hipotética NIP $\left(K_{N I P}=1 / R_{N I P}\right)$ e c) a curvatura da onda hipotética $\mathrm{N}\left(K_{N}=1 / R_{N}\right)$.

Para cada amostra $\left(x_{0}, t_{0}\right)$ na seção empilhada, i.e. a seção ZO a ser simulada, temos que determinar os três parâmetros i-CRS que produzem o melhor ajuste para um determinado evento de reflexão a ser simulado. Isto será feito através da análise de coerência (semblance, Taner e Koehler, 1969) ao longo da aproximação de tempos de trânsito i-CRS nos dados de cobertura múltipla.

Após a determinação do trio paramétrico do i-CRS procedemos à aplicação do empilhamento i-CRS. O empilhamento i-CRS consiste na soma das amplitudes dos traços sísmicos em dados de cobertura múltipla, ao longo da superfície definida pela aproximação de tempos de trânsito CRS implícita, que melhor se ajusta aos dados. O resultado é assinalado a pontos de uma malha pré-definida na seção AN. Como resultado tem-se a 
simulação de uma seção sísmica AN. Isto significa que para cada ponto da seção AN deve-se estimar o trio de parâmetros ótimos que produz a máxima coerência entre os eventos de reflexão sísmica.

\section{RESULTADOS}

\section{Modelo 1}

O primeiro modelo 2-D considerado neste estudo está constituído por duas camadas sob um semi-espaço e separadas por interfaces curvas e suaves (Figura 2).

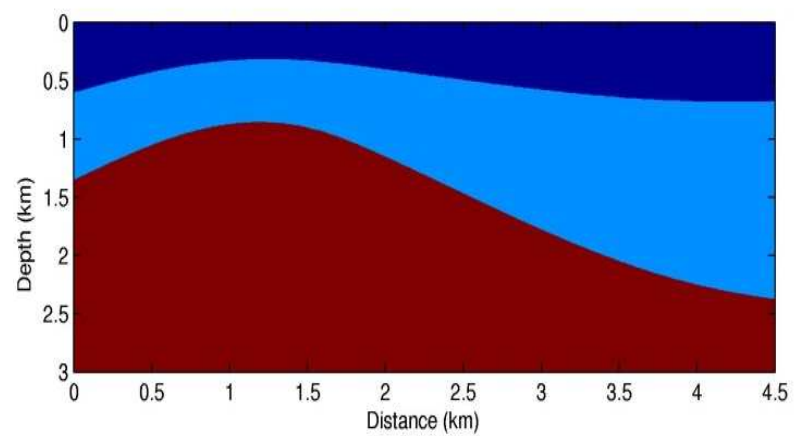

Figura 2 - Modelo 2-D constituído por duas camadas de velocidades iguais, sobre um meio-espaço com interface curva. Suas velocidades intervalares são de $2,5 \mathrm{~km} / \mathrm{s}, 3,0$ $\mathrm{km} / \mathrm{s}$ e $3,5 \mathrm{~km} / \mathrm{s}$, respectivamente.

Baseado neste modelo foi gerado o conjunto de dados sintéticos de reflexão de cobertura múltipla utilizando o algoritmo de traçamento de raio, SEIS88 (Cerveny e Psensik, 1988). Os dados não contêm ruído e foram gerados utilizando a configuração common-shot (CS) ou de tiro comum. O mínimo e máximo afastamento foram $0,6 \mathrm{~km}$ e $2,9 \mathrm{~km}$ respectivamente.

Foi considerada uma fonte tipo Gabor com uma frequência dominante de $50 \mathrm{~Hz}$ e uma intervalo de amostragem de tempo de $0,002 \mathrm{~s}$. A primeira fonte foi posicionada em $X_{S}=0,5 \mathrm{~km}$ e o primeiro geofone em $X_{G}=1,1 \mathrm{~km}$, sendo distribuídos 48 geofones com espaçamentos de $0,025 \mathrm{~km}$ entre eles. Consideramos 70 tiros com intervalos de $0,05 \mathrm{~km}$ cada.

As seções sísmicas ZO simuladas são apresentadas na Figura 3 e constam de 81 traços com intervalos entre os traços de $0,025 \mathrm{~km}$.
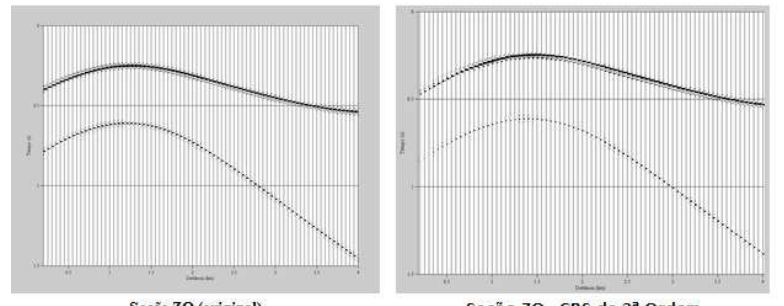

Seção ZO (original)

Seção ZO - CRS de $2^{\mathrm{a}}$ Ordem
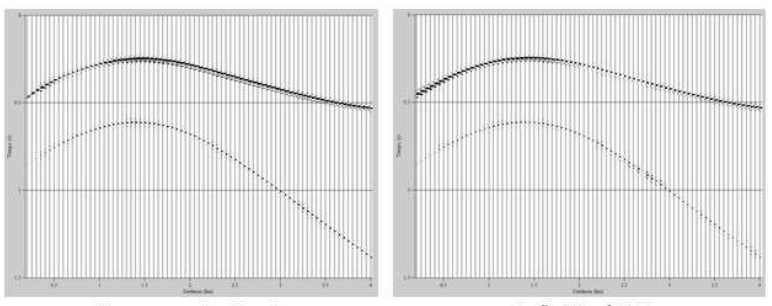

Secão ZO - CRS de $4^{\mathrm{a}}$ Ordem

Seção ZO - i-CRS

Figura 3 - Seções sísmicas ZO para o modelo 1: a) Traços sísmicos $Z O$ verdadeiros ou originais gerados com o software Seis88 a partir de dados sintéticos. b) Traços ZO simulados com a aproximação CRS de $2^{\text {a }}$ ordem. c) Traços $Z O$ simulados com a aproximação CRS de $4^{a}$ ordem. d) Traços $Z O$ simulada com a aproximação $i-C R S$.

Todas as seções simuladas apresentam um resultado satisfatório na simulação $\mathrm{ZO}$ de eventos de reflexões primárias.

Comparando a seção ZO original com as seções CRS $2^{\text {a }}$ e $4^{\text {a }}$ ordem e i-CRS (Figura 3), observamos em geral um bom desempenho do i-CRS quanto comparado com o empilhamento CRS ( $2^{\mathrm{a}}$ e $4^{\mathrm{a}}$. ordem). Desta forma, notase uma boa definição dos refletores e uma boa aproximação da seção original.

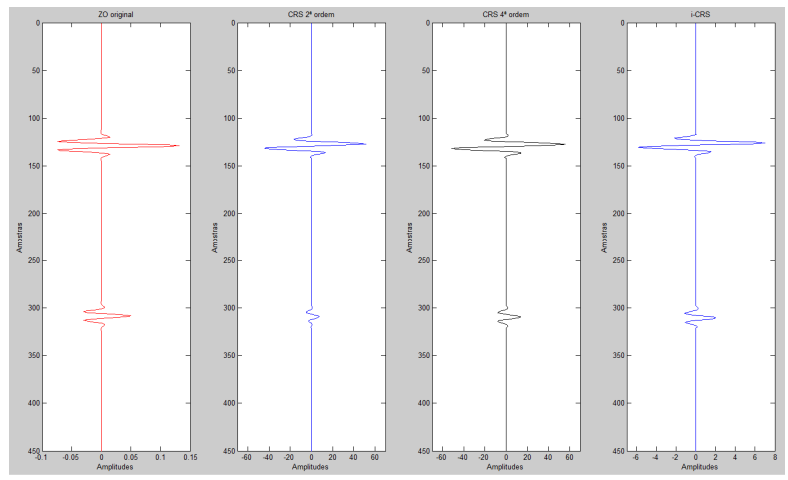

Figura 4 - Comparação do traço sísmico ZO original (modelo 1) com os traços $Z O$ simulados com as aproximações de tempos de trânsito CRS de $2^{\underline{a}}$ ordem, CRS de 4aㅡ ordem e i-CRS, respectivamente.

Na Figura 4 apresenta-se uma comparação entre o traço ZO original correspondente à coordenada ponto médio $x_{0}=1,15 \mathrm{~km}$, e os traços ZO obtidos a partir do 
empilhamento CRS de $2^{a}$ ordem, CRS de $4^{a}$ ordem e iCRS. O método i-CRS apresentou resultados satisfatórios na simulação da forma do traço ZO quando comparados com o CRS convencional e de $4^{\underline{a}}$ ordem.

\section{Modelo 2}

Para o próximo modelo 2-D neste estudo, foi considerado uma camada sobre um semi-espaço onde a interface tem a forma de um domo. Os tempos de trânsito para o modelo 2 (Figura 5) .
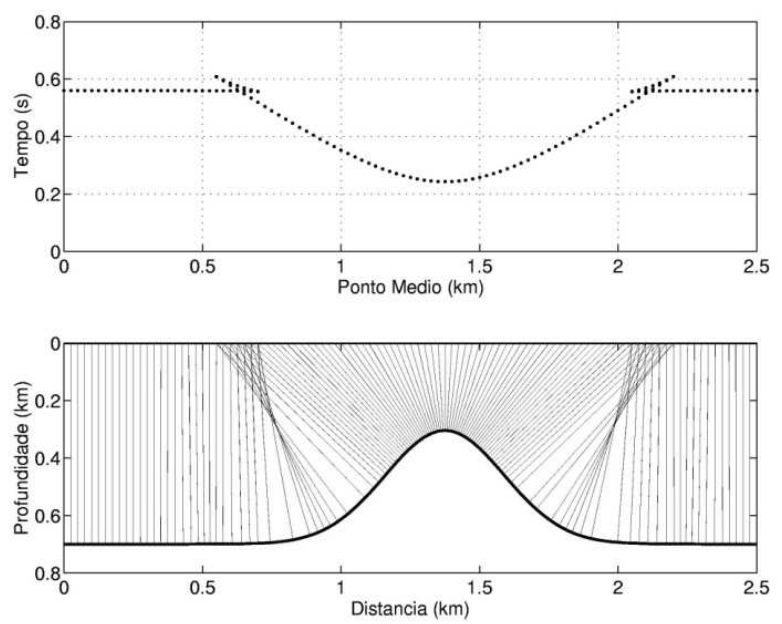

Figura 5 - Parte inferior: modelo tipo domo constituído por uma camada de velocidade constante de $2,5 \mathrm{~km} / \mathrm{s}$ e um semi-espaço infinito com uma velocidade de $3,0 \mathrm{~km} / \mathrm{s}$ respectivamente e seus correspondentes raios normais tipo ZO apresentando caústicas. Parte superior: tempos de trânsito ZO correspondentes aos anteriores raios normais.

Os dados não contêm ruído e foram gerados utilizando a configuração common-shot (CS) ou de tiro comum. O mínimo e máximo afastamento foram $0,6 \mathrm{~km}$ e 2,9 km respectivamente.

Foi considerada uma fonte tipo Gabor com uma frequência dominante de $50 \mathrm{~Hz}$ e uma intervalo de amostragem de tempo de $0,002 \mathrm{~s}$. A primeira fonte foi posicionada em $X_{S}=0,5 \mathrm{~km}$ e o primeiro geofone em $X_{G}=1,1 \mathrm{~km}$, sendo distribuídos 48 geofones com espaçamentos de $0,025 \mathrm{~km}$ entre eles. Consideramos 70 tiros com intervalos de $0,05 \mathrm{~km}$ cada. A seção sísmica de afastamento nulo a ser simulada é mostrada na Figura 6.4 e consta de 81 traços com intervalos de $0,025 \mathrm{~km}$.

Mais uma vez para verificar o desempenho do novo método em flancos íngremes, foram simuladas seções ZO a partir dos métodos de empilhamento CRS convencional de $2^{\underline{a}}$ e $4^{\mathrm{a}}$ ordem (Figura 6). Ao serem comparadas com a seção ZO original percebe-se um melhor desempenho na simulação dos traços ZO por parte do empilhamento i-CRS quando comparado com o empilhamento CRS de $2^{\mathrm{a}}$ e $4^{\mathrm{a}}$ ordem, respectivamente.

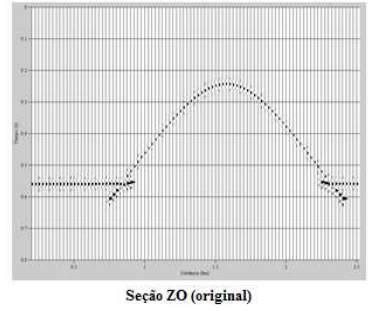

Seção ZO (original)

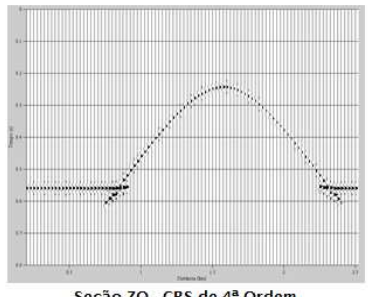

Seção ZO - CRS de $4^{\mathrm{a}}$ Orden

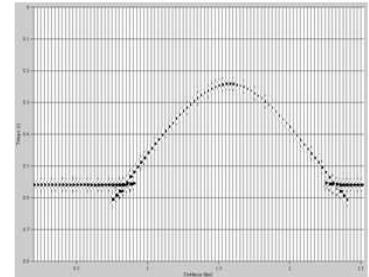

Seção ZO - CRS de $2^{\mathrm{a}}$ Ordem

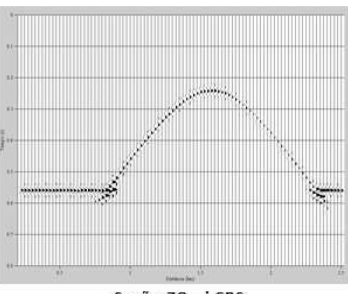

Seç̃o ZO-i-CRS
Figura 6 - Seções sísmicas ZO para o modelo 2: a) Traços sísmicos $Z O$ verdadeiros ou originais gerados com o software Seis88 a partir de dados sintéticos. b) Traços ZO simulados com a aproximação CRS de $2^{a}$ ordem. c) Traços $Z O$ simulados com a aproximação CRS de $4^{a}$ ordem. d) Traços $Z O$ simulada com a aproximação $i-C R S$.

Na Figura 7 apresenta-se uma comparação entre o traço ZO original correspondente à coordenada ponto médio $x_{0}=0,51 \mathrm{~km}$, e os traços sísmicos recuperados como produto do empilhamento i-CRS, CRS de $2^{\underline{a}}$ e $4^{\mathrm{a}}$ ordem.

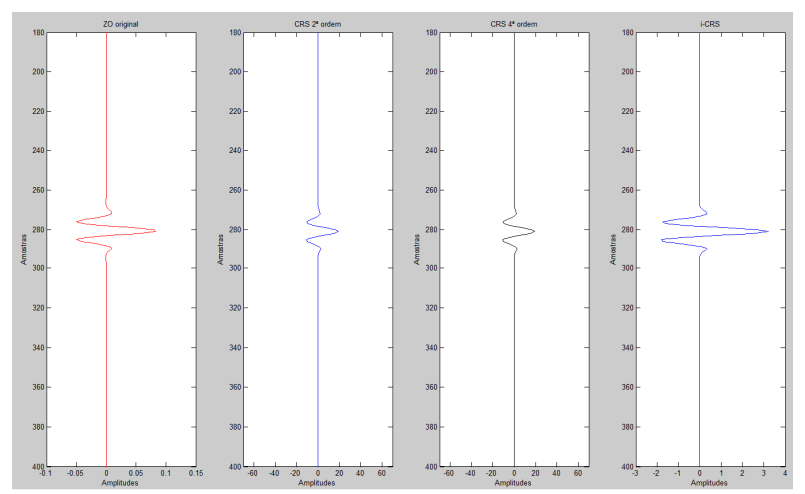

Figura 7 - Comparação do traço sísmico ZO original (modelo 2) com os traços $Z O$ simulados com as aproximações de tempos de trânsito CRS de $2^{a}$ ordem, CRS de 4를 ordem e i-CRS, respectivamente.

Observa-se na Figura 7 uma melhor recuperação na forma do traço ZO quando é aplicado o método i-CRS

\section{Conclusões}

Como consequência deste trabalho e analisando os resultados, conclui-se: 
A aproximação de tempos de trânsito i-CRS simula satisfatoriamente e coerentemente as seções com afastamento fonte-receptor nulo (ZO). Segundo os resultados obtidos podemos observar um bom posicionamento dos tempos de trânsito e uma boa visualização dos referidos eventos.

> Também podemos notar que o método i-CRS tem um bom desempenho para refletores bem curvos.

$>0$ método i-CRS apresenta-se como uma importante alternativa para simular seções ZO contendo reflexões primárias no caso de estruturas com flancos íngremes.

$>$ No que se refere à forma do pulso sísmico ZO simulado podemos concluir preliminarmente que o i-CRS é mais próximo da forma do pulso ZO original que as outras aproximações CRS.

$>$ Existem diferenças nos valores das amplitudes recuperadas na simulação $Z O$ por ambos métodos: CRS ( $2^{\mathrm{a}}$ e $4^{\mathrm{a}}$ ordem) e o i-CRS. Este métodos não recuperam a amplitude do traço ZO original.

\section{Agradecimentos}

Ao Programa Institucional de Bolsas de Iniciação Cientifica (PIBIC/UFPA) pela disponibilidade de bolsa de Iniciação Científica para o primeiro autor.

\section{Referências}

ČERVENÝ, V., PSENCIK, I., 1988. SEIS88, Ray Tracing Program Package.

DE BAZELAIRE, E. 1988. Normal moveout revisited inhomogeneous media and curved interfaces. Geophysics, 52, 143-157.

FOMEL, S., and V. GRECHKA, 2001, Nonhyperbolic reflection moveout of $P$ waves. An overview and comparison of reasons: Technical Report CWP-372, Colorado School of Mines.

HÖCHT, G., DE BAZELAIRE, E., MAJER, P. AND HUBRAL, P., 1999. Seismic and optics: hyperbolae and curvatures. Revita científica "Journal of Seismic Exploration", 12, p. 261-282.

JÄGER, R., MANN, J., HÖCHT, G. AND HUBRAL, P., 2001. Common Reflection Surface: Image and attributes. Geophysics, 66, 97-109.

LANDA, E., KEYDAR, MOSER, T. J., 2010. Multifocusing revisited - inhomogeneous media and curved interfaces. Geophysical Prospecting, 925-938.

LANDA, E., B. GUREVICH, KEYDAR, S., TRACHTMAN, P., 1999, Application of multifocusing method for subsurface imaging: Journal of Applied Geophysics, 42, 283-300.
MAYNE, W.H. 1962. Common reflection point horizontal data stacking techniques. Geophysics, 27(6):927-938.

MÜLLER, T., 1999. The common reflection surface stack method: Seismic imaging without explicit knowledge of velocity model. Germany, Karlsruhe University (Ph. D thesis).

SCHWARZ, B., VANELLE, C., KASHTA,. B., GAJEWKSI, D., 2012. i-CRS: application of a new multi-parameter stacking approach to complex media.

SCHWARZ, B., 2011. A new nonhyperbolic multiparameter stacking operator. Diploma thesis. Universität Hamburg (Germany), 104 p.

TANER, M. T., KOEHLER, F. 1969. Velocity spectradigital computer derivation and applications of velocity functions.Geophysics.

TYGEL, M.; MUELLER, T.; HUBRAL, P.; SCHLEICHER, J. 1997. Eigenwave based multiparametertraveltime expansions. In: ANNUAL MEETING OF SEG, 67.,Expanded abstracts, 1770-1773.

VANELLE, C., BOBSIN, M., SCHEMMERT, P., KASHTAN, B., GAJEWSKI, D., 2012. RSO - A new Multiparameter Stacking Operator for An/lsotropic media. $74^{\text {th }}$ EAGE Conference \& Exhibition incorportating SPE EUROPEC 2012. Copenhagen, Denmark.

VANELLE, C., KASHTAN, B., DELL, S., GAJEWSKI, D., 2010. A new stacking operator for curved subsurface structures (SPNA 3.2). 80 ${ }^{\text {th }}$ Ann. Int. Mtg. Soc. Expl. Geoph., Expanded Abstracts. 